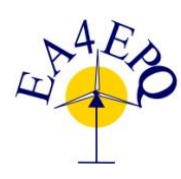

International Conference on Renewable Energies and Power Quality (ICREPQ'14)

Cordoba (Spain), $8^{\text {th }}$ to $10^{\text {th }}$ April, 2014

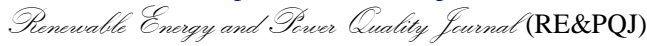

ISSN 2172-038 X, No.12, April 2014

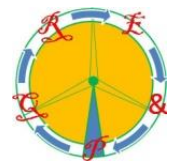

\title{
Study of defects in PV generators using image analysis techniques with Matlab
}

\author{
María José Pérez Regalado, Emilio Olías Ruiz, Plinio Jesús Pinzón. \\ Department of Electronics Technology \\ Carlos III University of Madrid \\ Madrid, Spain \\ emilio.olias@uc3m.es
}

\begin{abstract}
It is evidence that the useful life of the photovoltaic generator, as its correct operation, define the production and the profitability of a photovoltaic installation. There are several aspects that cause descents in the level of production of the generator and therefore losses in the installation. Based on it, it has developed numerous of both corrective and preventive maintenance that avoid these circumstances. Along the document there are revised the possible defects that the photovoltaic generator can have and the techniques of detection of the same ones. Inside the techniques of analysis and examination [1] we will center on the very concrete one: the visual inspection and its development by image analysis tools with Matlab. The techniques used are part of the tool "Image processing toolbox" that provides a finished set of algorithms of standard reference and graphic hardware to the prosecution, the analysis and the visualization of images and the development of complementary algorithms.
\end{abstract}

Across the employment and the adaptation of some of the included algorithms it will be claimed to approach a step more specializing in the conventional visual analysis used in the maintenance of the plants of photovoltaic solar energy.

Keywords-photovoltaic module, visual inspection, Matlab, image processing, defect.

\section{INTRODUCTION}

The photovoltaic generator is the responsible of the conversion of the incidental solar radiation in electrical energy. This fact turns it into the main device of the photovoltaic installation. Inside the initial inversion, the generator supposes between 50-70\%[2][3] of the cost of the installation and an anomalous functioning of the same one it goes so far as to cause between 5-10\% of losses.

It is frequent that the protocols proposed for the analysis and the optimization of the operation of the plant, often are not sufficient to guarantee a finished analysis of the state of the generator.
The methods of performance and the quality analysis proposed by the standards [4][5] they are focus on the functioning guarantee in the starting and its tests go from the detection of visual defects, tests of isolation, currents of escape up to evidences of open circuits. Nevertheless this turns out to be insufficient long-term, when the installation has been already submitted to the environmental conditions, typical degradation of the life time and conditions of functioning not always so uniform as it would turn out to be suitable.

The manufacturers provide recommendations both of installation and of maintenance to guarantee a few minimal requisites in the functioning. Typically the analyses are based on the defects supervision on the glass covering, penetration of moisture mistakes the encapsulated one, connections, pollution, or possibility of partial shadings.

In addition, it is common that there exists the inability to interrupt the service of the plant or to realize analysis of isolated form. For all this, at present there exist laboratories destined for the corrective maintenance and predictive of photovoltaic plants.

\section{FUNDAMENTALS OF DEFECTS IN PHOTOVOLTAIC GENERATOR}

There exists an important number of defects that can appear in the photovoltaic generator and that according to where they appear, which is its origin or frequency of declaration, the changes that cause in the curve $\mathrm{I}-\mathrm{V}$, in the operating temperature or other parameters typical of the photovoltaic module, can be considered to be more or less the reason cause decrease of useful life of the module.

The recognition of the different parts of the module, the types of defects that can appear, as well as of the main identification procedures, will be useful to us to identify those vulnerable parts of the generator and how we can approach its study. 
Regardless its technology or the type of semiconductor, usually the typical parts of a module are the glass covering, the encapsulant, the layer that contains the solar cells, the recovering of the backside and the frame. All this sealed of hermetic form by means of the pertinent production processes.

By means of this differentiation there appears next a table (Table I) that classifies the defects according to where or how they form and the possible internal after effects.

TABLE I. TIPES OF DEFECTS

\begin{tabular}{|c|}
\hline Encapsulating materials degradation \\
\hline $\begin{array}{c}\text { Breakage of glass } \\
\text { Dielectric breakdown } \\
\text { Failure of bypass diode } \\
\text { Discoloration of the encapsulant } \\
\text { Cracking } \\
\text { Delamination } \\
\text { Bubbles } \\
\text { Peels } \\
\text { Cracks } \\
\text { Yellowing and browning } \\
\text { Formation of grains in the weld metal } \\
\text { Burns in the solder joint } \\
\text { Losses on the glass powder } \\
\text { Degradation of the connection between cells }\end{array}$ \\
\hline Degradation of adhesion \\
\hline $\begin{array}{l}\text { Optical decoupling material } \\
\text { Decrease of the light transfer } \\
\text { Possibility of reverse bias } \\
\text { Increasing the temperature } \\
\text { Intrusion of fluids }\end{array}$ \\
\hline Moisture Penetration \\
\hline $\begin{array}{c}\text { Corrosion } \\
\text { Increased leakage currents } \\
\text { Increased electrical conductivity between materials } \\
\text { Oxidation of the mesh cell } \\
\text { Oxidation of terminals }\end{array}$ \\
\hline Degradation of semiconductor devices \\
\hline $\begin{array}{c}\text { Burns } \\
\text { Cell breakage } \\
\text { Increased resistance in the electric circuit } \\
\text { Formation of hot spots }\end{array}$ \\
\hline
\end{tabular}

Inside this list, there are more common defects, defects that can go unnoticed in production terms, and others that they can cause that the device gets damaged completely.

\section{PRINCIPAL DEFECTS DETECTION TECHNIQUES}

There are numerous skills of examination and photovoltaic maintenance that have emerge from the need for studies more exhaustive that guarantee the maximum performance.

\section{A. Thermography}

The temperature is one of the best indicators of mistake in any electromechanical installation. The thermography is an excellent technique to analyze distributions of this parameter by what it is used of habitual form in industrial and commercial environments.

By means of a thermographic inspection, we can visualize a series of bosses of temperature across which deducing the uniformity in the warming of the panel, defective cells, hot spots, etc.

It has the great advantage of being a noninvasive technique so, during the analysis of the device, operation will not be change.

\section{B. Electroluminiscence}

The devices used to realize this type of technique, are in charge of capturing the photonics radiation that takes place when the modules are in voltage, the electrons injected into the solar cell produce the filling of the available quantum wells transferring the excess by means of the photons emission. The intensity of the electroluminescence radiation is determined by the product of the electron and the concentration of the well, this radiation is infrared and therefore invisibly for the human eye. In normal conditions, this emission increases proportionally with the applied intensity.

Since the distance between the camera and the object of analysis is not established, this technique allows the analysis of independent cells and of finished modules; reaching if a more detailed analysis of particular areas will be necessary.

By means of this technique a big detail is revealed about structural defects, points of high series resistance, as well as micro fissures in the cells or other imperceptible cracks caused in the processes of manipulation of the module, which would be with visual techniques imperceptible. [6][7]

\section{RUV Resonance ultrasonic vibrations}

This is a technique based on the analysis of ultrasonic vibrations previously induced in the photovoltaic modules

The primary objective is the detection of cracks, fissures and micro fissures or even small holes that can unleash the formation of future cracks. The integration of this technique is realized in the proper production line. When there is a defect of this type, there is obtained the 
variation of the bandwidth and the resonance frequency like answer to the ultrasonic excitement of the waffle.

This technique is used of active form for the evaluation of photovoltaic cells of an autonomous way, although its use in already assembled photovoltaic modules is still limited. [8][9]

\section{Visual Inspection}

The visual inspection takes as a target to detect any present visual defect in the module. Before a few minimal conditions of 1000 lux, there have to be inspected the essential parts of the module: front, backside part, frame and junction box.

This method must not be considered to be anything trivial. Thanks to it is possible the detection of numerous types of such defects like bubbles, exfoliations, defective terminals, gratings etc can be detected. Nevertheless, the absence of standardization or regulation of a protocol of pursuit does that in any occasions a level of too rigorous analysis does not exist.

There are factors that can aggravate this fact as the inclusion of human errors, the existence of reflexes that could give place to wrong interpretations, defects of an invaluable size, etc. For it, is suitable to systematize, or to include more exhaustive analyses of the taken views to end by the mere perception of the human eye.

\section{EXPERIMENTAL APPROACH}

The samples used for the preliminary analysis correspond to photovoltaic modules of flat technology of polycrystalline silicon. These images have been obtained by a reflex camera of 18 megapixels, using the optical image stabilizer. The captures correspond to active parts of the modules, which present interest defects and which have been used like models of the analysis.

Based on the said thing, a preliminary study is carried out to analyze the obtained images, by means of some software of analysis of image with Matlab. For it, they have used or adapted some of the algorithms included inside "Image Processing Toolbox" that puts Matlab at the disposal of the user. The available graphic software, they allow to explore an image, examine a pixels region, fit the contrast, create outlines or histograms and manipulate interest regions. With the included algorithms degraded images can be restored, detect and measure functions, analyze forms and textures and fit color balances. [10]

The bases of the used methodologies, as well as the first approaches of the study appear next.

\section{A. Color-Based Segmentation using-K-means Clustering}

The method is based on a segmentation of the color of the image that it index in program by means of algorithm "imread" that it adapts for its later processing. In the first phase, the image is converted of RGB to Lab. The format RGB is a model of additive synthesis, what it implies that it is possible to reproduce any color for the miscellany of three primary colors, while Lab or CIELAB is a chromatic model used normally to describe all the colors that the human eye can perceive. Three parameters in the model represent the color luminosity $(\mathrm{L} *, \mathrm{~L} *=0$ black and $\mathrm{L}^{*}=100$ white), its position between red and green (a $*$, negative values indicate green while positive values indicate red) and its position between yellow and blue (b*, negative values indicate blue and positive values indicate yellow).

The digital conversion of images of the space RGB is given to Lab by the following expression [11] (ec.1):

$$
\left[\begin{array}{l}
L^{*}=116 f\left(Y / Y_{n}\right)-16 \\
a^{*}=500\left[f\left(X / X_{n}\right)-f\left(Y / Y_{n}\right)\right] \\
b^{*}=200\left[f\left(Y / Y_{n}\right)-f\left(Z / Z_{n}\right)\right]
\end{array}\right]
$$

The coordinates of the space of color CIEXYZ are X, $\mathrm{Y}, \mathrm{Z}$ and $\mathrm{Xn}, \mathrm{Yn}$ and $\mathrm{Zn}$, they would correspond to the value of the parameter of white color.

The conversion XYZ to RGB will be carried out later given by the following expression (ec.2):

$$
\left[\begin{array}{l}
X \\
Y \\
Z
\end{array}\right]=\left[\begin{array}{lll}
0.608 & 0.174 & 0.201 \\
0.299 & 0.587 & 0.114 \\
0.000 & 0.066 & 1.117
\end{array}\right]\left[\begin{array}{l}
R \\
G \\
B
\end{array}\right]
$$

The model of color Lab has been created to serve like an independent device or model that there is used like reference. The fundamental intention of using Lab, it is to produce a space of color that is perceptively more linear than other color spaces, that is to say, that before a change in the quantity in a value of color will reproduce a change almost of the same visual importance. Compared with $\mathrm{RGB}$, it is more rapid to do efficient color corrections in Lab. The fact that the luminosity is completely ignored in the channels "a" and "b", it does that it is much less sensitive to errors.

Although the number of possible numerical values for every pixel is less in Lab than in RGB, it is possible to index a top colors quantity in whole from the system Lab, not only colors that cannot be described by RGB but also colors that do not appear in the real world [12][13]. 
Later, thanks to the employment of the tool "k-means clustering" that, in a superficial way, can be described as a method of grouping and classification of a certain type of information, we will discriminate against those spaces or areas that could result from interest for the defects isolation.

In the figure 1 we can observe the results of one of the tests where, after realizing a previous visual recognition (figure 1a), discoloration can be observed along the module, as well as two breaks of cell in the average area of the panel. After this identification, the image is compiled in the application, segmenting it in a suitable number of colors to the regions distribution for the module that it wants to study. The primary objective is the isolation of areas that visually do not present defects of those that yes do it.

In the first approach, it segments in five differentiated regions, areas without defects (fig. 1b), total cell breakage (fig. 1c), partial cell breakage (fig. 1d), interconnections (fig. 1e) and discolored areas (fig. 1f).

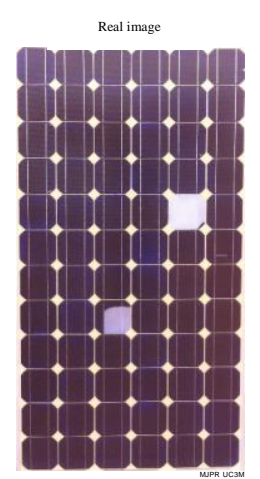

(a)

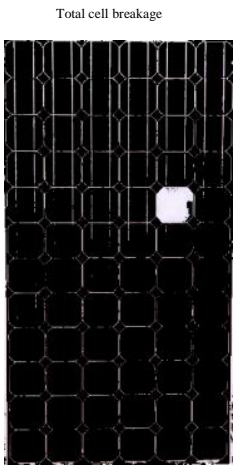

(c)

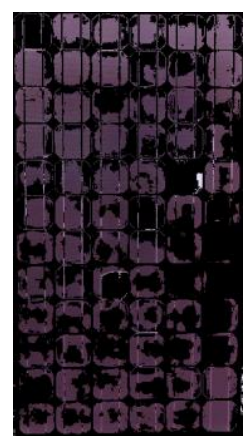

(b)

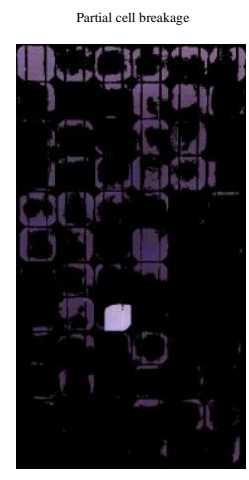

(d)

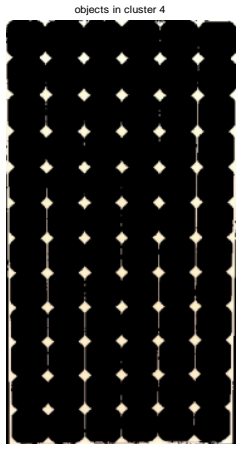

(e)

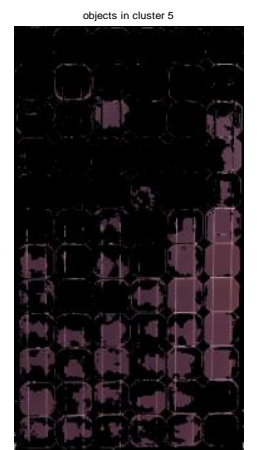

(f)
Figure 1. Sample processing with "Color-Based Segmentation usingK-means Clustering" (a)Real Image (b) areas without defects(c) total cell breakage (d) partial cell breakage (e) interconnections (f) areas discolored

Problems do not seem to appear in the isolation of the areas that do not present defects, as well as largely of the interconnection. In the figure 1 (d) "break of partial cell" there can be observed the areas that present discoloration of the encapsulant and that appear together with a break in the cell, likewise in the figure 1 (c) "break of entire cell" one of the breaks appears along with part of the interconnection that was not included in "interconnections" (figure1 (e)).

This is due to the fact that, in this case and for this typology of photovoltaic module, the interconnection presents a scale of color of character very similar to the break of cell therefore its discrimination turns out to be complex with the employment of this technique. In this case, the discrimination between certain areas would demand of a more sensitive processing.

Nevertheless, this technique would be adapted in case of absence of breaks or other defects of so clear character, which can be detected by other techniques of more effective form.

Based on it, the results that might be obtained by the sample of a module, which presents only discoloration, are studied bellow.

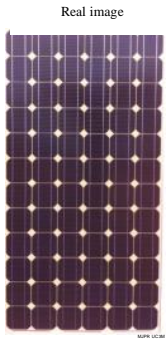

(a)

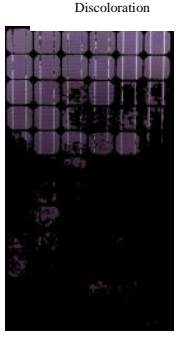

(b)

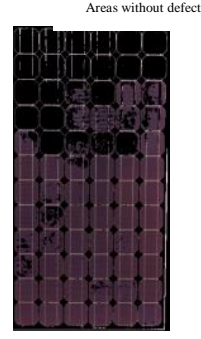

(c)
Figure 2. Analysis of discoloration with "Color-Based Segmentation using-K-means Clustering"(a) Real Image (b)“discoloration”(c) “areas without defects" 
An example of what has been mentioned might observe in the figure 2 , in the analysis of a module that presents discolorations, but he lacks more clear visual defects, we find an effective discrimination of the areas of interest, as it has already been commented.

\section{B. Correcting Non uniform ilumination}

By means of this technique it is possible to correct the lack of uniformity that exists in the captures of the photovoltaic module that are realized in field. It is common that during the solar exposure, the light that affects on the module presents lacking in uniformity, and it can be projected of irregular form in some areas, giving place to wrong interpretations.

In the first phase of the processing, the lighting of the image is estimated and later it normalizes. In the second phase it is possible to turn it into a binary image and to discriminate against objects that present a certain number of pixels.

On having realized the prosecution of image, we manage to improve the contrast avoiding reflexes, and darkened areas, contributing this way more definition to the affected parts and therefore facilitating its analysis. This corrective tool can be considered to be a preprocessing, to index-link images better defined in a later processing.

\section{Measuring Regions in Grayscale Images.}

The conversion is carried out by means of the command "regionprops", a very useful function for the morphologic prosecution of an image. Thanks to the same one, it is possible to take into account the number of objects of the capture and to locate its position inside the same one.

Thus the classified regions can measure themselves and separate for scales of color, in such a way that a defect might isolate itself in question.

This skill would present a major utility to isolate such clear defects like the breaks of cell.

In addition to it, it is possible to take into account the typical deviation of the pixels with regard to a reference point, what would be useful to take into account the discoloration grade, or to identify small areas that would go unnoticed for the human eye.

In the figure 3 presented next, it can be observed an example of analysis of the typical deviation in a photovoltaic cell that presents an entire break.

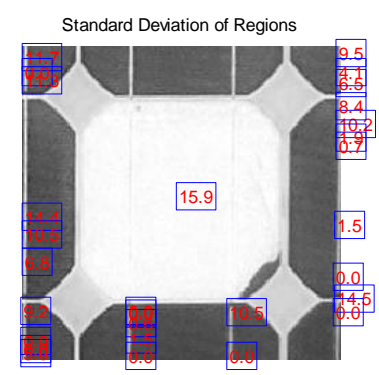

Figure 3. Color deviation using "Measuring Regions in Grayscale Images."

Although it can be considered to be an effective method, it does not contribute entire coverage to all the defects that want to be studied, it is by it that it is considered to be more opportune to center the development on other more adaptable algorithms for the user.

\section{Adaptation of the algorithm "Color Based Segmentation using the Lab Color Space”}

The objective of the method is to identify the different existing colors in the implemented image, to realize an analysis of the values of color by means of CIELAB that allows quantifying the existing visual differences.

The fundamental difference with regard to the method used first place, is that across the algorithm a small region of the sample is chosen for every color that wants to be discriminated, this sample will be used to classify properly every pixel.

The classification will be carried out across the Euclidean distance (ec.3) between the pixel that will be studied (P) and the pixel taken as model. (Q)

$$
d_{E}(P, Q)=\sqrt{\left(p_{1}-q_{1}\right)^{2}+\left(p_{2}-q_{2}\right)^{2}+\ldots+\left(p_{n}-q_{n}\right)^{2}}=\sqrt{\sum_{i=1}^{n}\left(p_{i}-q_{i}\right)^{2}}
$$

For example, if the distance between a pixel and the marker of red color is the smallest, of that time the pixel would be labeled like a red pixel.

Originally, this algorithm contains a color trace for every pixel; it is able to separating regions of color previously established in a model of sample. In order to realize a more exhaustive analysis, the samples of color, which are given by a coefficients matrix, are modified according to the studied tonality. Thus it is possible to establish the patterns of color that would show a typical defect.

In the analyzed samples there were identified previously the areas that wanted to be discriminated and take into account. 
In the figure 4 we can observe the example of submitting to this processing to one of the samples, where they have discriminated the interconnections, the areas with discoloration, the areas that do not present affection and the broken cells.

To the procedure there joins an algorithm, which takes into account the interest areas, labeling them properly.

The first tests try to realize effective discriminations of the defects for later to be related to electrical tests that provide some approach to us in the relation with the power losses.
Interconnections $(8,07 \%)$
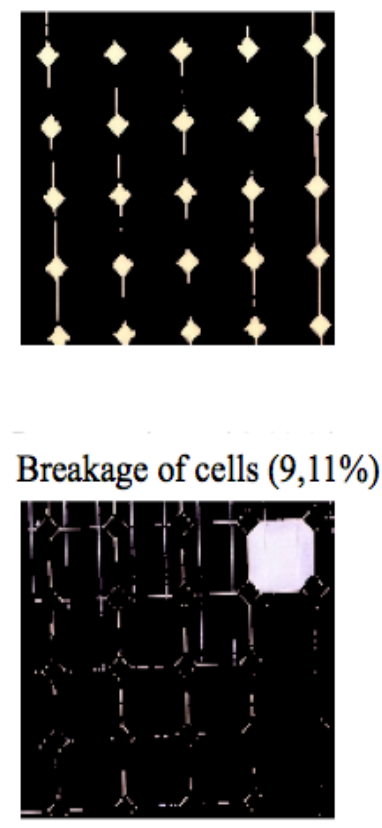

Discolorations $(10,3 \%)$

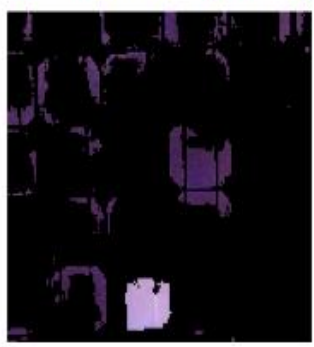

Healthy $(72,5 \%)$

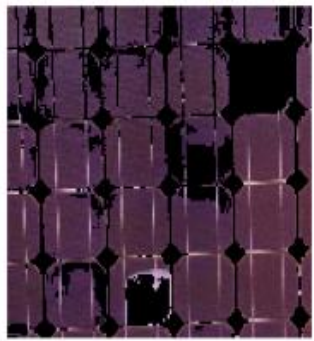

Figure 4. Results of processing a sample of the photovoltaic module using "Color Based Segmentation using the Lab Color Space”

\section{CONCLUSIONS}

Incorporating techniques of analysis of image for computer, we can omit part of the errors that would join to the examination.

The use of these techniques contributes major precision, the possibility of analysis of individual and detailed form, and the accounting of these defects of more rigorous form.

\section{ACKNOWLEDGMENT}

This work was supported by the NUMANCIA-2 project. Project Code: S2009/ENE-1477 funded by the Regional Government of Madrid (Spain).

\section{REFERENCES}

[1] E. Kaplani "Detection of degradation effects in fieldaged $\mathrm{c}-\mathrm{Si}$ solar cells through IR thermography and digital image processing" Mechanical Engineering Department, T.E.I. of Patras Greece, 2012.

[2] J.Coello, J.L. Galindo, M.Carames, R. Carreño.'Quality control during the supply of PV Modules: Fundamental key to guarantee the profitability of PV Installations" Enertis Solar, 2008.

[3] Stefan mau, Thomas Krametz, Wolfgang Jahna, Hubert Fechner "Quality testing for PV-modules according to standards and performance control for supporting manufacturing" arsenal research, Business Unit Renewable Energy Technologies Faradaygasse. 19th EUPVSEC, 2004.

[4] IEC 61215 "Crystalline silicon terrestrial photovoltaic (PV) modules. Design qualification and type approval Reference" (Ed. 2.0) 2005.

[5] IEC 61646 "Thin-film terrestrial photovoltaic (PV) modules. Design qualification and type approval" (Ed. 2.0) 2008.

[6] T. Fuyuki. "Photographic surveying of minority carrier diffusion length in polycrystalline silicon solar cells by electroluminescence." 2006.

[7] K. Bothe. Electroluminescence imaging as an in-line characterisation tool for solar cell production. 21st European Photovoltaic Solar Energy Conference, Dresden, German. 2006.

[8] Yu. Emirov, A. Belyaev, D. Cruson, I. Tarasov, A. Kumar ,H. Wu, S. Melkote and S. Ostapenko "Pinhole detection in $\mathrm{Si}$ solar cells using resonance ultrasonic vibrations, Ultrasonic Technologies, Inc., Wesley Chapel, FL USA, University of South Florida, Tampa, FL USA Georgia Institute of Technology, Atlanta, GA, USA ,2011.

[9] W Dallas, O Polupan and S Ostapenko, "Resonance ultrasonic vibrations for crack detection in photovoltaic silicon wafers" University of South Florida, Nanomaterials and Nanomanufacturing Research Center, 4202 East Fowler Ave, Tampa, FL 33620, USA, 2007

[10] González, Rafael C. "Digital image processing using MATLAB”, ED. Pearson/Prentice Hal , 2004

[11] Mr. Vivek Singh Rathore, Mr. Messala Sudhir Kumar, Mr. Ashwini Verma "Colour Based Image Segmentation Using L*A*B* Colour Space Based On Genetic Algorithm" International Journal of Emerging Technology and Advanced Engineering,2012.

[12] Yufa Zhang, Zunyang Liu, Zirong Wang, Dabin Yu "Influence of Color Difference Formulas on Image Dominant Colors Extraction" State Key Laboratory of Pulsed Power Laser Technology Electronic Engineering Institute, Fifth International Symposium on Computational Intelligence and Design China, 2012.

[13] Michael Wirth and Denis Nikitenko, "The effect of colour space on image sharpening algorithms" University of Guelph School of Computer Science, Canadian Conference Computer and Robot Vision. 2010 . 